\title{
Modulation of macrophage activation status by bisphosphonates and breast cancer cells.
}

Sofia Sousa ${ }^{1}$, Jukka Mönkkönen ${ }^{1}, J o r m a ~ M a ̈ a ̈ t t a ̈{ }^{1,2}$

${ }^{1}$ School of Pharmacy, Faculty of Health Sciences, University of Eastern Finland

2 Institute of Biomedicine, Department of Cell Biology and Anatomy, University of Turku, Finland

\section{Introduction}

Tumour stromal macrophages differentiate into tumour associated macrophages (TAMs), with characteristics resembling the immunosuppressive M2 polarization instead of the pro-inflammatory M1. TAMs have a central role in promoting tumour vascularization, cancer cell dissemination and suppression of anti-cancer immune response. Cancer cell dissemination leads to metastasis formation which e.g. in breast cancer often happens in bone marrow. We have studied the in vitro modulation of that macrophage polarization by bisphosphonates (BPs) and breast cancer cell conditioned medium (CM)

Diagram 1. BPs mechanisms of action.

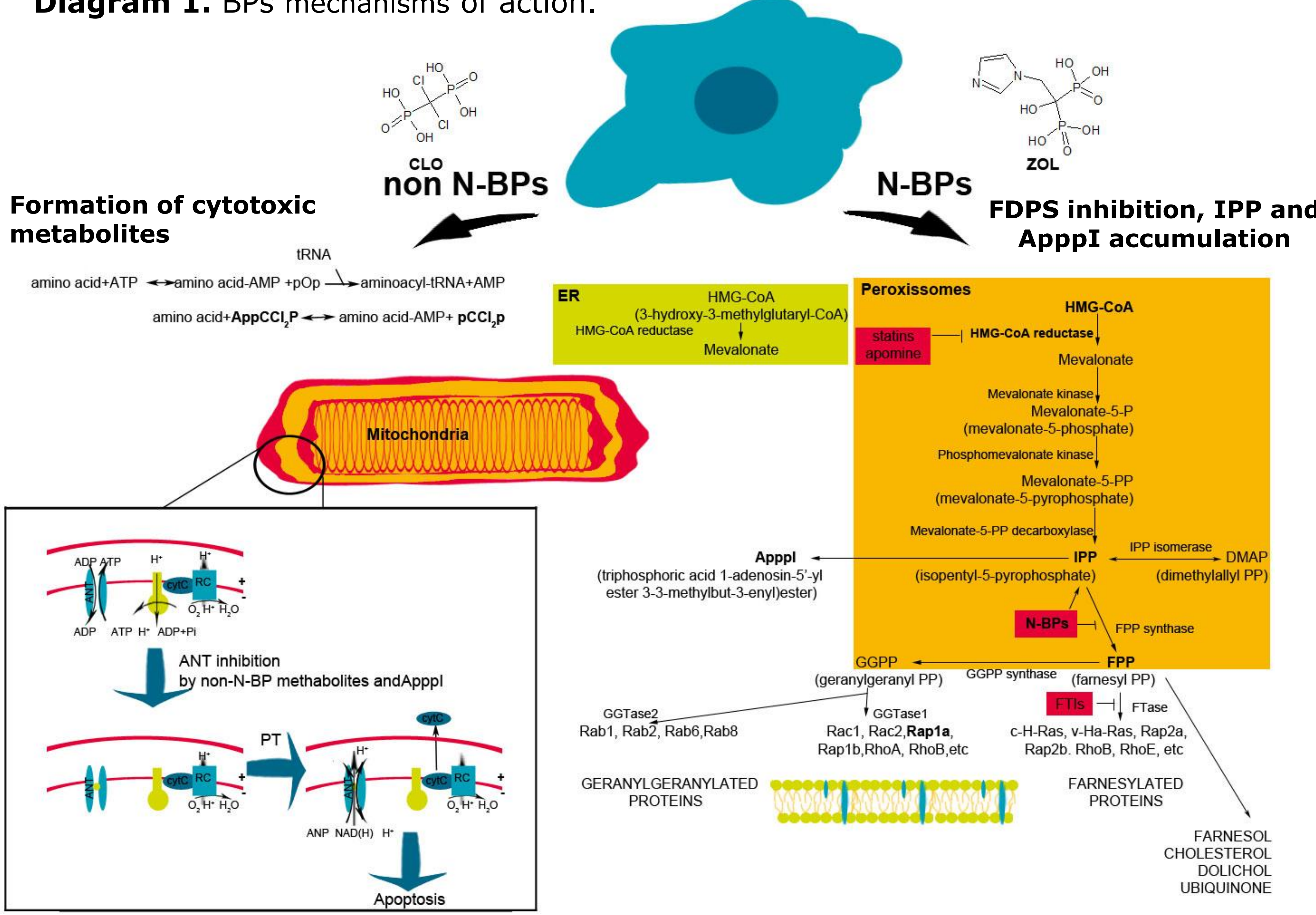

Methods

The murine macrophage cell line 3774 was treated for $48 \mathrm{~h}$ with semi-logarithmic concentrations of free and liposome encapsulated clodronate (CLO) and zoledronate (ZOL) and cell viability was assessed.

$\mathrm{J} 774$ cells were cultured for $72 \mathrm{~h}$ in the presence of $50 \% \mathrm{CM}$ either from the murine breast cancer cell line 4T1 or murine fibroblast cell line 3T3. After conditioning cells were further stimulated with $10 \mathrm{ng} / \mathrm{mL}$ LPS for $24 \mathrm{~h}$.

The same macrophage cell line was conditioned for $72 \mathrm{~h}$ with $50 \% 4 \mathrm{~T} 1 \mathrm{CM}$, treated for $24 \mathrm{~h}$ with free or liposome encapsulated CLO (CLO-LIP) and ZOL (ZOL-LIP) and further stimulated with $10 \mathrm{ng} / \mathrm{mL}$ LPS for $24 \mathrm{~h}$.

Supernatants were colected for multiplex ELISA, Griess assay and zymography.

IPP, ApppI and $\mathrm{AppCCl}_{2} \mathrm{p}$ were determined by HPLC-MS in acetonitrile/water extracts from BP treated $\mathrm{J774}$ cells. For western blot analysis total cell lysates were used.

Results

Fig. 1 A-Growth inhibition and cytotoxicity effects of free and liposome encapsulated BPs. BIPP, ApppI and $\mathrm{AppCCl}_{2} \mathrm{p}$ accumulation. C-Protein prenylation inhibition by $\mathrm{ZOL}$

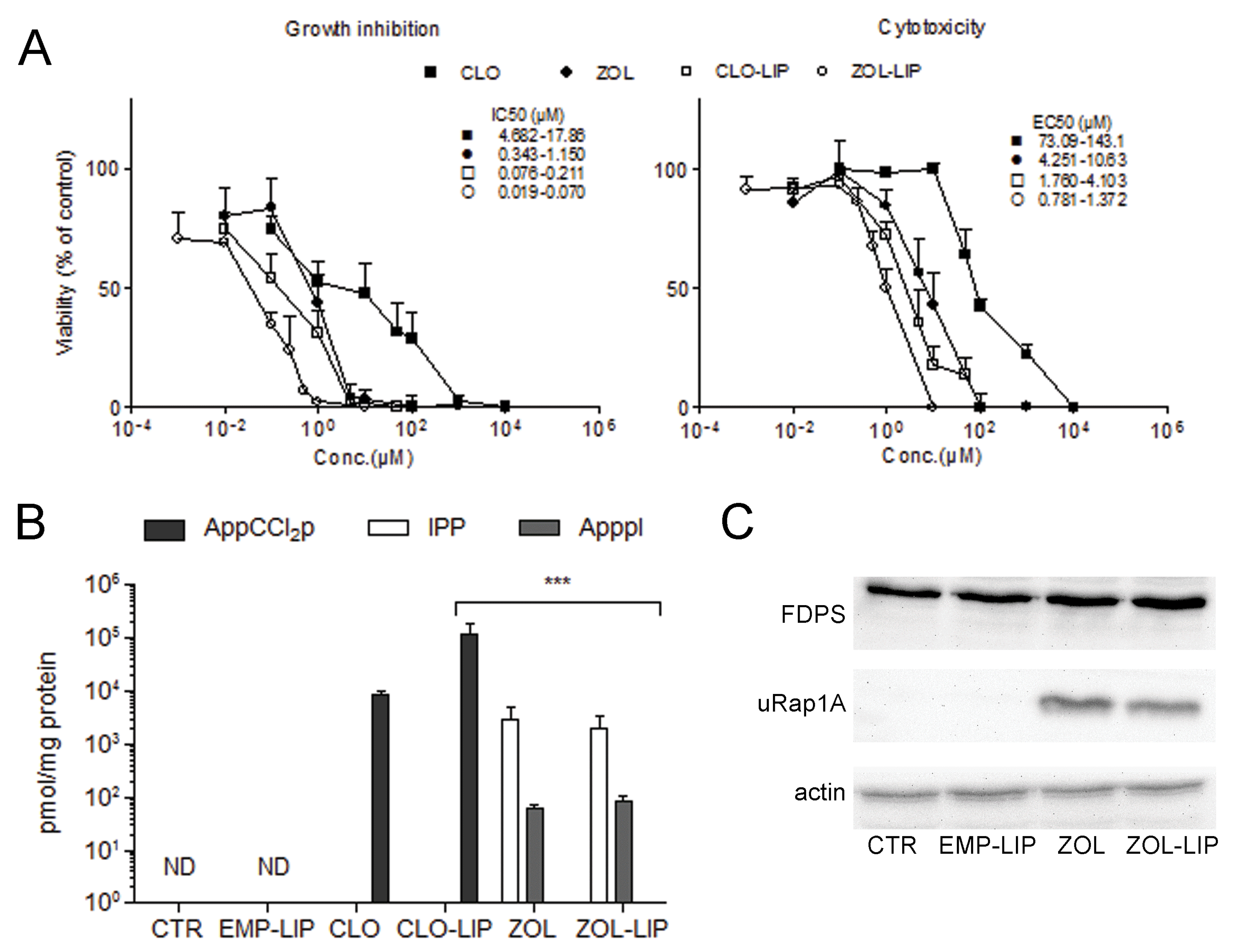

All data presented is unpublished.

Work supported by EC's $7^{\text {th }} \mathrm{FP}$, grant agreement 264817

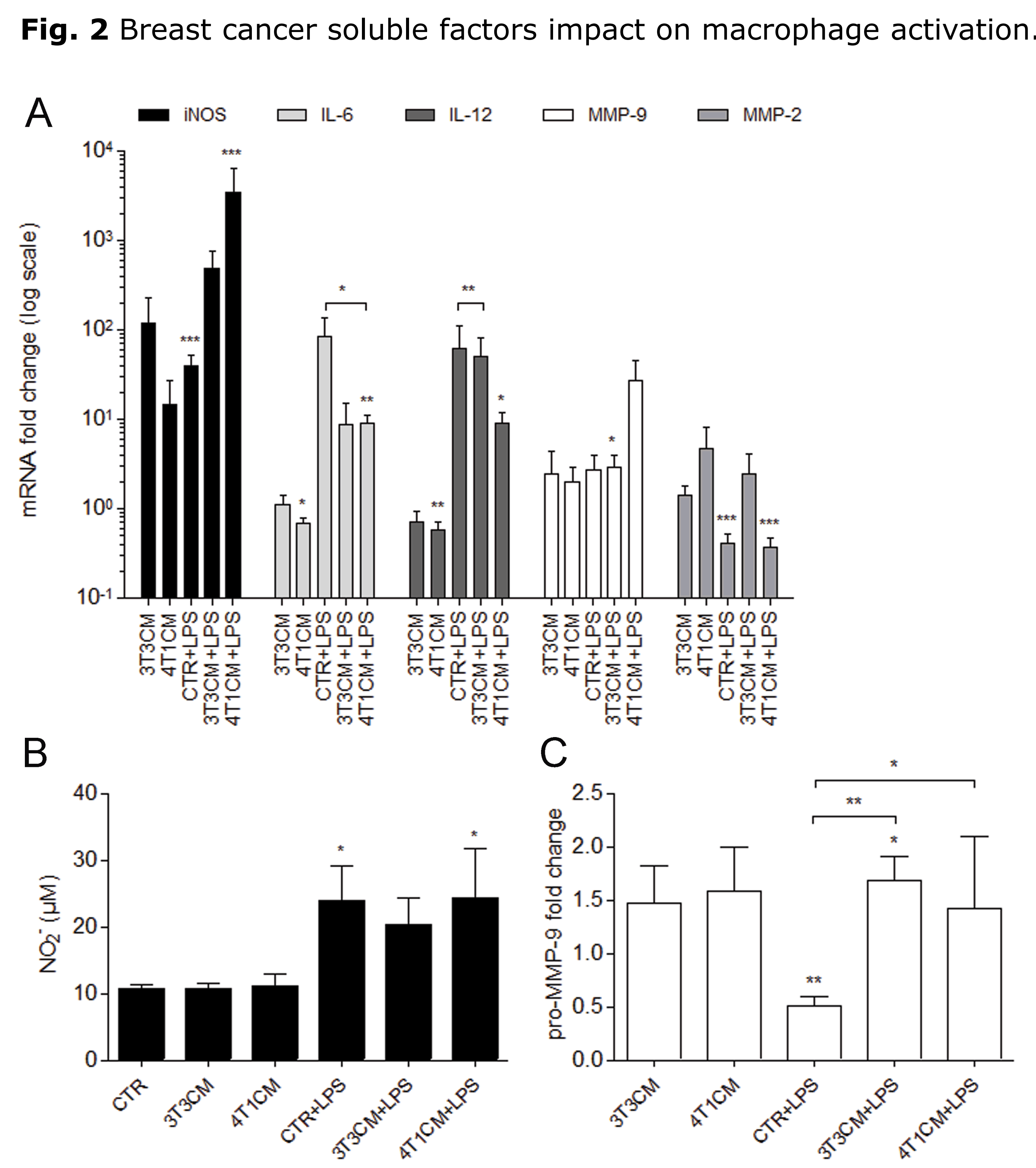

Fig. 3 Free and liposome encapsulated BPs impact on macrophage activation.

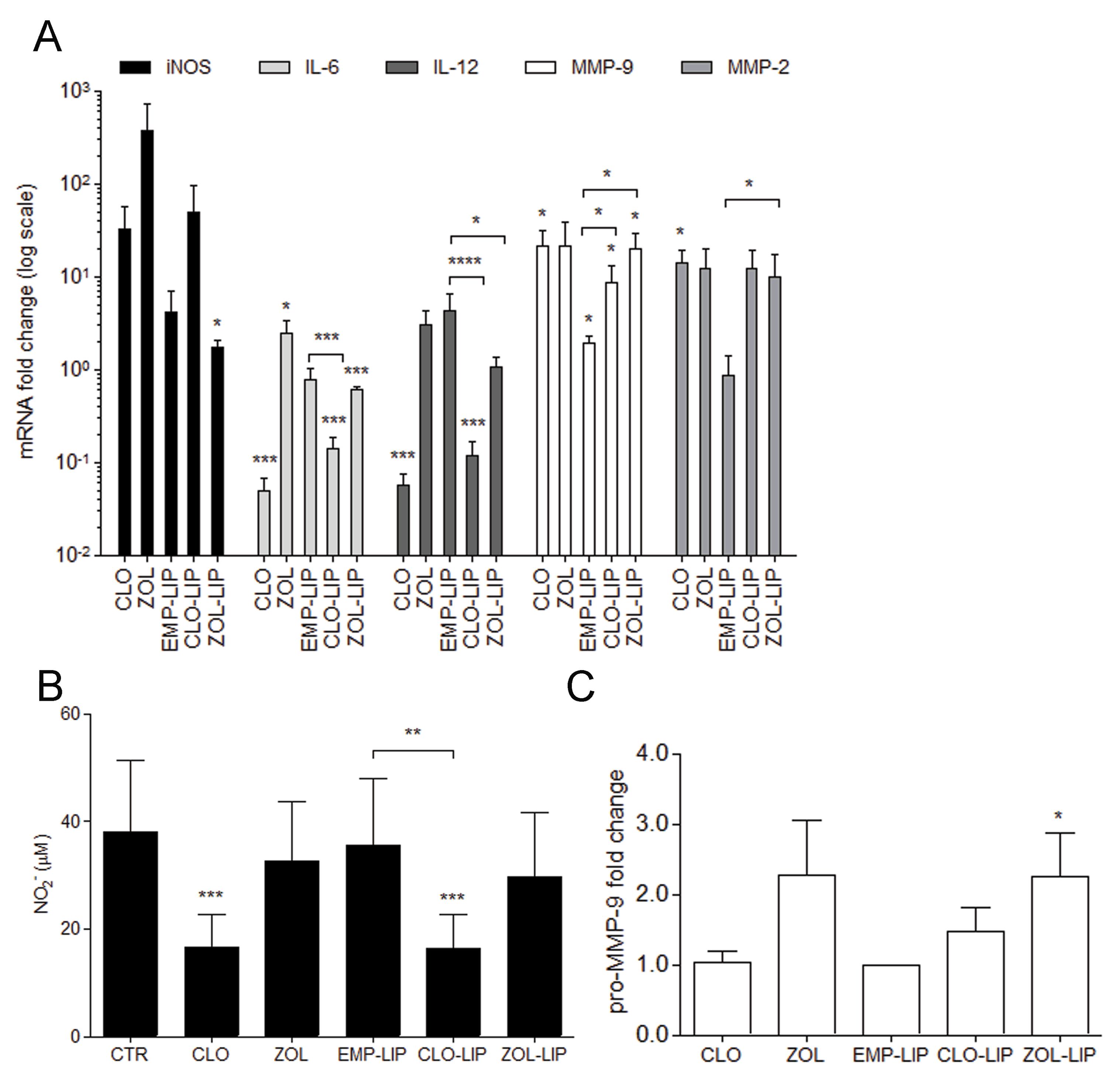

Conclusions

Breast cancer cells secreted factors modulate macrophage activation, increasing IL-6, IL-12 and MMP-9 production upon LPS stimulus. However macrophages seem less responsive to LPS when conditioned by breast cancer factors.

- Liposome encapsulation increases BPs potency, and in vivo may be used to overcome the bone seeking properties of these drugs.

- CLO decreases NO, IL- 6 and IL-12 production by macrophages and ZOL increases MMP-9.

- Further studies are needed to clarify the potential clinical relevance of these distinct macrophage modulation by N-BP and non-N-BP. The choice of which BP to use should be context and aim dependent. If the objective is to reduce inflammation a non-N-BP should be used. However if aiming to increase macrophage aggressiveness without decreasing inflammation, a N-BP might be beneficial. 